\title{
Study of Opportunistic routing in ad-hoc network under different network scenario using ns-2.
}

\author{
Manisha Ahirrao, Prof.Beena Ballal \\ ${ }^{1}$ Vidyalankar Institute of Technology,Electronics and Telecommunication Engineering \\ ${ }^{2}$ Vidyalankar Institute of Technology, Electronics and Telecommunication Engineering
}

\begin{abstract}
An ad-hoc routing protocol is a convention, or standard, that controls how nodes decide which way to route packets between computing devices in a mobile ad hoc network. Since the nodes can move freely, then the nodes mobility pattern became an important in case of MANET. In ad-hoc networks, nodes are not familiar with the topology of their networks. Instead, they have to discover it. The basic idea is that a new node may announce its presence and should listen for announcements broadcast by its neighbors. Each node learns about nodes nearby and how to reach them. In this work we proposed the behavior of reactive protocol under different network scenario. The transmission rate is calculated for different network scenario keeping the pause time constant .It is concluded that it does not create extra traffic for communication, it gives higher reliability of data delivery and uses a destination sequence number which keeps an up to date path to the destination node
\end{abstract}

KEYWORDS: Routing, Ad-Hoc network, Scenario, NS2.

\section{INTRODUCTION}

Traditional approach for opportunistic routing protocol generates poor quality path which have low availability, low reliability, high energy consumption. This happens because of quality of a wireless channel fluctuates with time \& routing protocol is unaware of these fluctuations. For this a concept of opportunistic routing has been proposed. The concept of opportunistic routing is very powerful and well suited to sensor networks where there are significant disruptions to node availability. However, there are other non opportunistic approaches. In particular, we would like to compare the performance of opportunistic routing with geographic routing since they are similar in many ways. They are both based on a view that in dynamically changing environments such as sensor networks, trying to find static and optimized routes does not make sense. Both of them require no routing tables beyond the knowledge of neighbors and thus are very scalable for large networks. In fact, opportunistic routing is in many ways an extension of geographic routing; since it explicitly acknowledges the transient nature of the channel and node availability. Hence it is important to understand when it becomes profitable to move from geographic to opportunistic routing.

This paper deals with investigating that question by conducting a systematic performance evaluation. It explores the performance of opportunistic routing for different node densities, node pause time and node speed. Transition points are found using simulations for each of the above parameters to identify the communication. A second contribution is to identify optimal operation points for various scenarios for opportunistic routing. The rest of the paper is organized as follows. Section 2 discusses related works followed by a short description of the AODV protocol in Section 3. The simulation setup is described in Section 4 and the results are presented in Section 5. Finally, the paper concludes with Section 6.

\section{MANET}

A mobile ad-hoc network is a collection of wireless mobile hosts that forms a temporary network without the requirement of any standalone infrastructure or centralized administration. Mobile Ad-hoc networks are self-organizing and self-configuring multi hop wireless networks where, the structure of the network changes dynamically. This is mainly due to the mobility of the nodes. The nodes in the network not only acts as hosts but also as routers that route data to/from other nodes in network. The mobile ad-hoc networks with no infrastructure behave as a wireless networks, if a destination node is out of range of a source node transmitting packets; a routing procedure is always needed to find a path so as to forward the packets appropriately between the source and the destination. In the case of ad-hoc networks, each node must be able to forward data for other nodes.

\subsection{Classification of routing protocols}

Routing protocols are classified depending on what the routers tell each other and how they use the information 
Study of Opportunistic routing in ad-hoc network under different network scenario using ns-2.

to form their routing tables. They are Distance vector protocols and Link state protocols[2][8].

\subsubsection{Distance Vector Protocols}

In distance vector protocols, each router sends the information to reach to the destination over the internetwork neighboring routers, The routers sends two pieces of information first, the router tells, how far it thinks the destination is and secondly, it tells in what direction (vector) to use to get to the destination. When the router receives the information from the others, it then create a table of destination addresses, distances and associated neighboring routers, and from this table it select the shortest route to the destination[7]. The best example for this is the routing information protocol (RIP).

\subsubsection{Link-State Protocols}

In link state protocols, a router provide information about the topology of the network it does not have the information about the destination [2]. This information consist of the network segments and links that are attached to that particular router along with the state of the link i.e., whether the link is in active state or the inactive state. This information is flooded throughout the network and then every router in the network then builds its own picture of the current state of all the links in the network.

\subsubsection{Topology based}

Topology based approaches, which are further divided into two subcategories:

Reactive, Proactive and Hybrid use information about links to forward the packets between nodes of the network. Hybrid routing protocols are actually combination of feature of both type of protocols, which aggregates all nodes into various areas in topology structure[3].Prominent protocols of this category are Proactive DSDV, Reactive AODV, DSR. and Hybrid zone routing protocol(ZRP).

\subsubsection{Position based routing protocols.}

Position-based routing (e.g. LAR) requires some information about the physical or geographic positions of the participating nodes [6]. In this protocol the routing decision is not based on a routing table but at each node the routing decision is based on the positions of its neighboring nodes and the position of the destination node.

\section{AD HOC ON DEMAND DISTANCE VECTOR (AODV)}

AODV [5] discovers routes on an as needed basis via a similar route discovery process .In AODV, whenever a source node has to communicate with a destination node such that it has no routing information in its table, it first initiates route discovery process. The node broadcasts a route request (RREQ) packet to all its neighbors[1].The route request packets contains source address, source sequence number, broadcast ID, destination address, destination sequence number and hop count. The source address and broadcast ID uniquely identifies a RREQ. The source sequence number is for maintaining the freshness of information about the reverse route to the source. The destination sequence number specifies how fresh a route to the destination must be before it can be accepted by the source. If a neighbor knows the route to the destination, it replies with a route reply control message RREP that propagates through the reserve path. Otherwise, the neighbor will rebroadcast the RREQ until an active route is found or the maximum number of hops is reached.

\section{SIMULATION DESIGN}

The simulation experiment is carried out in LINUX (FEDORA 6). The detailed simulation model is based on network simulator-2 (ver-2.34)[4] .The basic configuration is that our testing is in a $700 * 700$ square with variable node numbers. The traffic sources are CBR (constant bit rate), 512-byte as data packets, sending rate is 4 pkts /second. To use CBR, since bit rate vary will make the data packets traffic load unpredictable, which situation we do not want it happen. The number of sources we can use are 10, 20, 30, 40 and so on. The node pause time is set 20 seconds and it can be varied depending on the application. Once the destination is reached, another random destination is targeted after a pause. The protocols are evaluated for packet delivery ratio, pause time , and speed at varying node densities (10 to 40) 


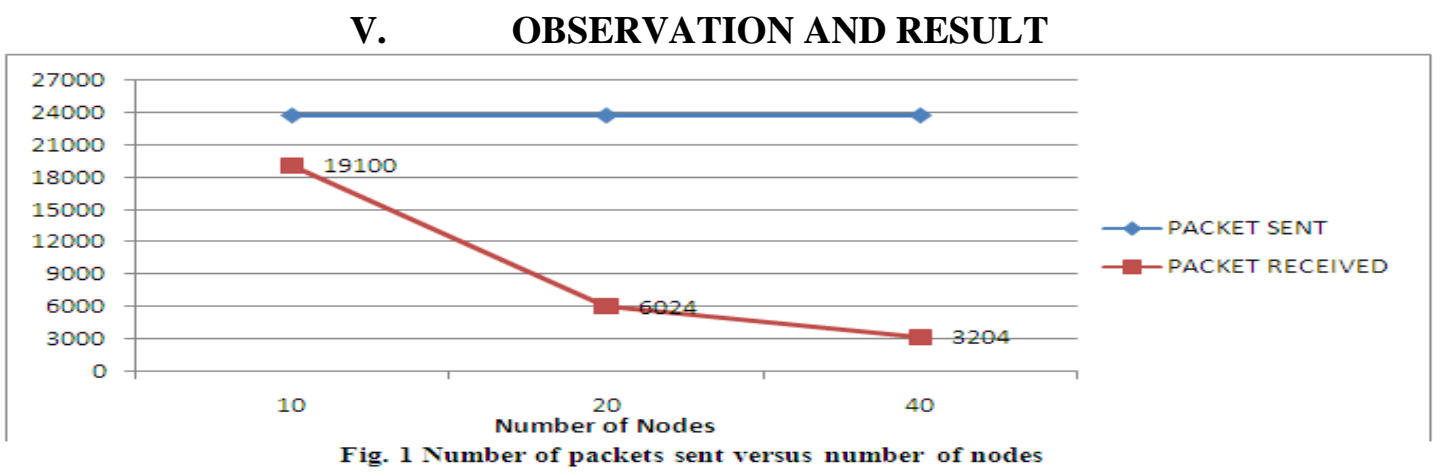

\section{CONCLUSION AND FUTURE WORK}

In this paper, performance of routing protocols AODV is evaluated for An ad hoc networks under different network scenarios. The AODV protocol is studied with different node density for various metrics. From Fig. 1 it is found that as the no of nodes get increased the packet takes more time to reach to the destination. For future work, we want to study the impact of transmission range on the performance of the Routing protocols. Future work will also include the evaluation of other position based routing protocols. There are several advantages due to its simple implementation It does not create extra traffic for communication, it gives higher reliability of data delivery and uses a destination sequence number which keeps an up to date path to the destination node.

\section{REFERENCES}

[1]. Krishna Gorantala, Routing Protocols in Mobile Ad-hoc Networks, Master's Thesis in Computing Science,Sweden,June 15, 2006

[2]. Geetam S. Tomar, Position Based Routing for Wireless Mobile Ad Hoc Networks, IJSSST, Vol. 10, No. 1

[3]. Samyak Shah, Amit Khandre, Mahesh Shirole and Girish Bhole, Performance Evaluation of Ad Hoc Routing Protocols Using NS2 Simulation, IJCSET |January 2012| Vol 2, Issue 1,787-791

[4]. Ian D. Chakeres, Elizabeth M. Belding-Royer, AODV Routing Protocol Implementation Design , IOSR Journal of Engineering, 2(2), 2012, 112-116.

[5]. NS-2, The ns Manual (formally known as NS Documentation) available at http: //www. isi.edu/nsnam/ns/doc.

[6]. Mubashir Husain Rehmani, A Tutorial on the Implementation of Ad-hoc On Demand Distance Vector (AODV)

[7]. Protocol in Network Simulator (NS-2),Sidney Doria, and Mustapha Reda Senouci _Version 1,28th June 2009

[8]. Guoyou He, Networking Laboratory ,Helsinki University of Technology, Destination-Sequenced Distance Vector (DSDV) Protocol, www.netlab.tkk.fi/opetus/s38030/k02/Papers/03-Guoyou

[9] Zehua Wang, Opportunistic and Cooperative Forwarding in Mobile Ad-hoc Networks with Light-Weight Proactive Source Routing, A thesis submitted to the School of Graduate Studies in partial fulfillment of therequirements for the degree of Master of Engineering, University of Newfoundland,September 2011 